\title{
BOTTOM SPIN VALVE BASED ON THE ORDERED Ni-Fe-Mn ANTIFERROMAGNETIC PHASE
}

\author{
I. V. Blinov*, M. A. Milyaev, V. V. Popov \\ M.N. Miheev Institute of Metal Physics, Ural Branch of the Russian Academy of Sciences, \\ 18 S. Kovalevskoy St., Ekaterinburg, 620108, Russian Federation \\ *Corresponding author. E-mail: blinov@imp.uran.ru \\ Address for correspondence: 18 S. Kovalevskoy St., Ekaterinburg, 620108, Russian Federation \\ Tel.: +7 (343) 3783592
}

\begin{abstract}
Magnetic properties of bilayers based on the $\mathrm{Ni}-\mathrm{Fe}-\mathrm{Mn}$ antiferromagnetic phase are studied, as well as magnetic and magnetoresistive properties of a bottom spin valve (SV) with a Ni-Fe-Mn antiferromagnet as a pinning layer. A technique for the fabrication of bottom spin valves with enhanced thermal stability and improved hysteretic characteristics has been developed. Specimens were made by DC magnetron sputtering and electron-beam evaporation on glass (Corning) and single-crystalline sapphire (1012). For the preparation of bottom SV, the specimens were etched in a PlasmaPro NGP 80 RIE Oxford Instruments reactive ion-plasma etching device. The magnetoresistance of the as-fabricated $\mathrm{Al}_{2} \mathrm{O}_{3} / \mathrm{Ni}-\mathrm{Fe}-$ $\mathrm{Mn} / \mathrm{Co}_{90} \mathrm{Fe}_{10}(5.5 \mathrm{~nm}) / \mathrm{Cu}(3.6 \mathrm{~nm}) / \mathrm{Co}_{90} \mathrm{Fe}_{10}(5.5 \mathrm{~nm}) / \mathrm{Ta}(5 \mathrm{~nm})$ spin valve is $\Delta R / R_{\mathrm{s}}=3.8 \%$.
\end{abstract}

Keywords: unidirectional anisotropy, spin valve, ordered $\mathrm{Ni}-\mathrm{Fe}-\mathrm{Mn}$ antiferromagnetic phase.

DOI: $10.17804 / 2410-9908.2017 .6 .057-063$

\section{References}

1. Giant Magnetoresistance: Basic Concepts, Microstructure, Magnetic Interactions and Applications / I. Ennen, D. Kappe, T. Rempel, C. Glenske, A. Hotten // Sensors. - 2016. Vol. 16, no. 6. P. 904. - DOI: 10.3390/s16060904

2. Giant magnetoresistance of (001)Fe/(001)Cr magnetic superlattices / M. N. Baibich, J. M. Broto, A. Fert, F. Nguyen van Dau, F. Petroff, P. Etienne, G. Creuzet, A. Friederich, J. Chazelas // Phys. Rev. Lett. - 1988. - Vol. 61, no. 7. - P. 2472-2475. - DOI: 10.1103/PhysRevLett.61.2472

3. Coehoorn R. Giant Magnetoresistance and Magnetic Interactions in Exchange-Biased SpinValves // Handbook of magnetic materials / ed. by K. H. J. Buschow. - Amsterdam : Elsevier B.V., 2003. - Vol. 15. - P. 1-199.

4. Magnetoresistive sensors / P. P. Freitas, R. Ferreira, S. Cardoso, F. Cardos // J. Phys.: Condens. Matt. - 2007. - Vol. 19, no. 16. - P. 165221-1-21. - DOI: 10.1088/0953-8984/19/16/165221

5. Ustinov V. V., Milyaev M. A., Naumova L. I. Giant magnetoresistance of metallic exchange-coupled multilayers and spin Valves // Physics of Metals and Metallography. - 2017. Vol. 118, no. 13. - P. 38-97.

6. Formation of Ordered NiFeMn Antiferromagnetic Phase in Permalloy/Manganese Bilayers in the Course of Thermomagnetic Treatment / I. V. Blinov, T. P. Krinitsyna, A. V. Korolev, S. A. Matveev, N. K. Arkhipova, M. A. Milyaev, V. V. Popov, V. V. Ustinov // Physics of Metals and Metallography. - 2014. - Vol. 115. - P. 335-341. - DOI: 10.1134/S0031918X14040036

7. Svalov A. V., Vas'kovskii V. O., Yarmoshenko Yu. M. Production and study of spin valve structures on the permalloy film base // Fiz. Met. Metalloved. - 1995. - No. 79. - P. 270-273. 
Подана в журнал: 13.12 .2017

УДК 669.1'24'74:539.216.2:537.621

DOI: $10.17804 / 2410-9908.2017 .6 .057-063$

\title{
СПИНОВЫЙ КЛАПАН С НИЖНИМ РАСПОЛОЖЕНИЕМ УПОРЯДОЧЕННОЙ АНТИФЕРРОМАГНИТНОЙ ФАЗЫ Ni-Fe-Mn
}

\author{
И. В. Блинов", М. А. Миляев, В. В. Попов \\ Федеральное государственное бюджетное учреждение науки \\ Институт физики металлов имени М.Н. Михеева Уральского отделения Российской академии наук, \\ ул. С. Ковалевской, 18, Екатеринбург, Российская Федераиия \\ *Ответственный автор. Электронная почта: blinov@imp.uran.ru \\ Адрес для переписки: ул. С. Ковалевской, 18, 620990, Екатеринбург, Российская Федерация \\ Телефон: +7 (343) 378-35-92
}

В работе исследованы магнитные характеристики бислоев с антиферромагнитной (АФ) упорядоченной фазой $\mathrm{Ni}-\mathrm{Fe}-\mathrm{Mn}$, а также магнитные и магниторезистивные свойства спинового клапана с закрепляющим АФ слоем Ni-Fe-Mn. Разработана методика приготовления спинового клапана с нижним расположением антиферромагнитной фазы $\mathrm{Ni}-\mathrm{Fe}-\mathrm{Mn} \mathrm{c}$ повышенной термостабильностью и улучшенными гистерезисными характеристиками. Слоистые образцы изготавливались при помощи напылительной системы магнетронного напыления MPS-4000-C6 (Ulvac) и технологии электронно-лучевого напыления на сверхвысоковакуумной установке Varian (США) на стеклянные (Corning) и монокристаллические сапфировые подложки $\mathrm{Al}_{2} \mathrm{O}_{3}$ (1012). Ионное травление образцов, предназначенное для удаления поверхностного слоя, выполнялось на установке реактивного ионно-плазменного травления PlasmaPro NGP 80 RIE Oxford Instruments. Магнитосопротивление приготовленного спинового клапана $\mathrm{Al}_{2} \mathrm{O}_{3} / \mathrm{Ni}-\mathrm{Fe}-\mathrm{Mn} / \mathrm{Co}_{90} \mathrm{Fe}_{10}(5,5 \mathrm{~nm}) / \mathrm{Cu}(3,6 \mathrm{~nm}) / \mathrm{Co}_{90} \mathrm{Fe}_{10}(5,5 \mathrm{~nm}) / \mathrm{Ta}(5 \mathrm{~nm}$ составляет $\Delta R / R_{\mathrm{s}}=3,8 \%$.

Ключевые слова: однонаправленная анизотропия, спиновый клапан, упорядоченная фаза $\mathrm{Ni}-\mathrm{Fe}-\mathrm{Mn}$.

\section{1. Введение}

Современные магниторезистивные наноматериалы, состоящие из ультратонких слоев магнитных и немагнитных металлов, обладают эффектом гигантского магнитосопротивления (ГМС), благодаря чему имеют широкое применение в различных технических приложениях [1]. Гигантский магниторезистивный эффект, обнаруженный в 1988-1990 гг. в сверхрешетках, полученных методами молекулярно-лучевой эпитаксии и магнетронного напыления, достигал 10-80 \% в поле насыщения $\left(H_{\mathrm{s}}\right)$ напряженностью в десятки кЭ при 4,2 K [2].

В 1991 г. был разработан новый тип наноструктуры - спиновый клапан. В простейшем случае спиновый клапан состоит из двух ферромагнитных (ФМ) слоев, разделенных немагнитной прослойкой, и антиферромагнитного (АФ) слоя [3]. При этом в одном из ФМ слоев, связанном с АФ слоем, в результате обменного взаимодействия на интерфейсе ФМ/АФ формируется однонаправленная магнитная анизотропия. Петля магнитного гистерезиса бислоя ФМ/АФ оказывается смещенной по оси магнитного поля. Смещение центра петли гистерезиса относительно нуля характеризуется полем смещения $\left(H_{\mathrm{ex}}\right)[3]$.

Для спиновых клапанов характерно резкое изменение электрического сопротивления в малых магнитных полях. Большие величины магнитосопротивления $(5 \ldots 15 \%)$ и чувствительности $(1 \ldots 5$ \%/Э) делают спиновые клапаны удобным материалом для применения в устройствах микроэлектроники [4, 5]. 
Эффективная работа спиновых клапанов определяется, прежде всего, высоким значением $H_{\mathrm{ex}}$ с отношением $H_{\mathrm{c}} / H_{\mathrm{ex}}<1$ ( $H_{\mathrm{c}}-$ коэрцитивная сила ФМ слоя, связанного с АФ). Важным для работы клапана является также высокое значение температуры блокировки $\left(T_{\mathrm{b}}\right)$. При повышении температуры обменное взаимодействие на границе ферромагнетикантиферромагнетик уменьшается и разрушается при приближении к температуре Нееля $\left(T_{\mathrm{N}}\right)$. При этом поле смещения петли магнитного гистерезиса бислоя ФМ/АФ становится равным нулю при критической температуре $-T_{\mathrm{b}}$. Как правило, $T_{\mathrm{b}}$ всегда меньше температуры Нееля массивного АФ [3]. Величина сдвига петли магнитного гистерезиса и температурная стабильность характеристик спинового клапана в значительной степени зависят от используемого антиферромагнитного материала. Для повышения температурной стабильности спиновых клапанов необходимо использовать антиферромагнетики с температурой Нееля значительно превышающую комнатную температуру. Необходимость повышения температурной стабильности обусловлена как требованиями к эксплуатационным характеристикам изделий на базе спиновых клапанов, так и ограничениями, связанными с технологическим циклом изготовления сенсорных элементов методами литографии. В последнем случае ограничения связаны с тем, что в процессе литографических операций используются температуры более $200{ }^{\circ} \mathrm{C}$. Если температура блокировки для АФ будет ниже используемых технологических температур, то в процессе изготовления сенсоров анизотропные свойства спинового клапана изменятся, что приведет к ухудшению функциональных характеристик конечных изделий на их основе.

Одним из перспективных материалов для закрепляющего слоя в спиновых клапанах является упорядоченная фаза $\mathrm{Ni}-\mathrm{Fe}-\mathrm{Mn}$. Ранее нами установлен механизм формирования упорядоченной антиферромагнитной $\mathrm{Ni}-\mathrm{Fe}-\mathrm{Mn}$ фазы при термомагнитной обработке бислоев марганец-пермаллой [6]. При отжиге марганец проникает в пермаллой по границам кристаллитов, а затем диффундирует от границ в приграничные объемы матрицы. При этом сплошной антиферромагнитный слой между слоями не образуется. Формирование упорядоченной антиферромагнитной фазы $\mathrm{Ni}-\mathrm{Fe}-\mathrm{Mn}$ в бислоях $\mathrm{Ni}_{77} \mathrm{Fe}_{23} / \mathrm{Mn}$ приводит к высокой величине обменного смещения $\left(H_{\mathrm{ex}}=380 Э, J_{\mathrm{ex}}=0,27\right.$ эрг $\left./ \mathrm{cm}^{2}\right)$ с температурой блокировки $270{ }^{\circ} \mathrm{C}$ [6]. Полученные характеристики сопоставимы с характеристиками дорогостоящего сплава IrMn [4]. Упорядоченная АФ фаза Ni-Fe-Mn перспективна для использования в спиновых клапанах вследствие высокой температуры блокировки и высоких значений поля обменного смещения.

Цель работы заключается в разработке методики, позволяющей создавать спиновые клапаны с высокой термостабильностью, включающие упорядоченную антиферромагнитную фазу $\mathrm{Ni}-\mathrm{Fe}-\mathrm{Mn}$.

\section{2. Образцы и методика эксперимента}

Образцы изготавливались по технологии электронно-лучевого напыления на сверхвысоковакуумной установке Varian (США). Толщины слоев контролировались с помощью кварцевого толщиномера. Также была использована оптимизированная технология напыления наноструктур при помощи напылительной системы магнетронного напыления MPS4000-C6 (Ulvac). Толщины слоев определялись по известной скорости напыления, определенной экспериментально для каждого распыляемого материала. Определение скорости напыления материалов производилось с помощью оптического профилометра Zygo NewView 7300 по известному времени напыления и измеренной толщине пленки.

Для магнетронного напыления использовались мишени аттестованного состава. Элементный состав пленок контролировался при помощи рентгеновского микроанализатора на базе растрового электронного микроскопа с автоэмиссионным катодом FEI Inspect F, оборудованного приставкой Genesis APEX 2 EDS. 
Измерение магнитных и магниторезистивных свойств образцов было проведено на метрологически аттестованном оборудовании. Исследование магнитных характеристик приготовленных пленок было выполнено на автоматизированном вибрационном магнитометре ABM-1 в автоматическом режиме в диапазоне магнитных полей \pm 5 кЭ при комнатной температуре. Магнитосопротивление определялось четырехконтактным методом с погрешностью 0,1 \%. Намагниченность насыщения образцов определялась с погрешностью 3 \%.

Ионное травление образцов, предназначенное для удаления поверхностного слоя, выполнялось на установке реактивного ионно-плазменного травления PlasmaPro NGP 80 RIE Oxford Instruments.

Термомагнитная обработка наноструктур проводилась при давлении $10^{-4}$ Па в постоянном магнитном поле 2 кЭ, приложенном в плоскости образца при температуре $260{ }^{\circ} \mathrm{C}$.

\section{3. Результаты и обсуждение}

Для создания спинового клапана с нижним расположением упорядоченной АФ фазы $\mathrm{Ni}-\mathrm{Fe}-\mathrm{Mn}$ нами была разработана оригинальная методика со следующей последовательностью операций:

- приготовление электронно-лучевым напылением двухслойных пленок марганецпермаллой;

- формирование упорядоченной АФ фазы $\mathrm{Ni}-\mathrm{Fe}-\mathrm{Mn}$ путем отжига в магнитном поле бислоя марганец/пермаллой;

- ионное травление приготовленного образца марганец/пермаллой для удаления поверхностного слоя толщиной 30 нм;

- последующее магнетронное напыление на образец после ионного травления слоистой структуры, состоящей из ферромагнитных слоев сплава $\mathrm{Co}_{90} \mathrm{Fe}_{10}$ разделенных немагнитным слоем меди;

- термомагнитная обработка приготовленного образца при температуре выше температуры блокировки.

Для получения упорядоченной антиферромагнитной фазы использовался разработанный ранее метод, описанный в [6]. Состав наноструктуры и режим термомагнитной обработки выбирали таким образом, чтобы в образце $\mathrm{Al}_{2} \mathrm{O}_{3} / \mathrm{Ni}_{77} \mathrm{Fe}_{23}\left(5\right.$ нм)/Mn(50 нм)/ $\mathrm{Ni}_{77} \mathrm{Fe}_{23}(30$ нм)/Ta(5 нм) получить максимальное поле смещения петли магнитного гистерезиса с высокой температурой блокировки. Сразу после напыления образец был отожжен в магнитном поле напряженностью 2 кЭ при температуре $260{ }^{\circ} \mathrm{C}$ в течение 4 ч без разгерметизации вакуумной камеры. После этого наноструктуру переносили в установку для ионного травления для удаления поверхностного слоя. Продолжительность ионного травления выбирали таким образом (20 и 30 мин), чтобы гарантированно удалить загрязненный поверхностный слой и оставить в образце ферромагнитную часть и упорядоченную АФ фазу $\mathrm{Ni}-\mathrm{Fe}-\mathrm{Mn}$, которые необходимы для формирования однонаправленной анизотропии и возникновения смещенной петли гистерезиса в ФМ слое $\mathrm{Co}_{90} \mathrm{Fe}_{10}$, который напыляется на поверхность образца после травления. На рис. 1 показано распределение концентраций элементов в отожженном образце, полученное при помощи Оже-электронной спектроскопии. Это исследование позволило определить картину диффузионного перемешивания на различных стадиях отжига и сформулировать модель зернограничной диффузии марганца в пермаллой [6].

Из рис. 1 следует, что упорядоченная АФ фаза $\mathrm{Ni}-\mathrm{Fe}-\mathrm{Mn}$ существует приблизительно при толщине слоя до 50 нм. 


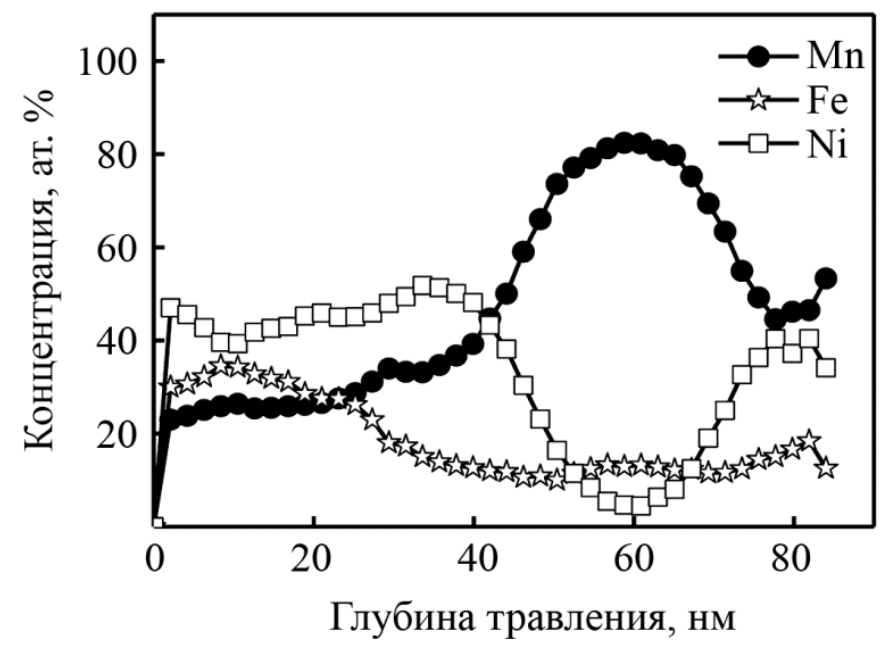

Рис. 1. Распределение концентраций элементов по глубине в образце $\mathrm{Al}_{2} \mathrm{O}_{3} / \mathrm{Ni}_{77} \mathrm{Fe}_{23}(5 \mathrm{Hм}) / \mathrm{Mn}(50 \mathrm{Hм}) / \mathrm{Ni}_{77} \mathrm{Fe}_{23}(30 \mathrm{нм}) / \mathrm{Ta}(5 \mathrm{м})$ после отжига при $260{ }^{\circ} \mathrm{C}$ в течение 4 ч

Для определения оптимального режима ионного травления отожженного образца варьировалось время травления от 20 до 30 мин. Подходящими магнитными свойствами обладает образец, для которого использовалось ионное травление продолжительностью 20 мин. При этом с отожженного образца $\mathrm{Al}_{2} \mathrm{O}_{3} / \mathrm{Ni}_{77} \mathrm{Fe}_{23}(5$ нм $) / \mathrm{Mn}(50 \mathrm{Hм}) / \mathrm{Ni}_{77} \mathrm{Fe}_{23}(30$ нм $) / \mathrm{Ta}(5$ нм $)$ удаляется слой толщиной приблизительно 30 нм. Далее в тексте такая созданная структура обозначена как $\mathrm{Al}_{2} \mathrm{O}_{3} / \mathrm{Ni}-\mathrm{Fe}-\mathrm{Mn}$.

После ионного травления образец $\mathrm{Al}_{2} \mathrm{O}_{3} / \mathrm{Ni}-\mathrm{Fe}-\mathrm{Mn}$ был помещен в камеру загрузки магнетронной установки, оснащенную радиочастотным магнетроном, позволяющим проводить очистку подложек методом обратного распыления. Время радиочастотного распыления - 4 мин, при этом толщина удаленного слоя составляет 5 нм. Слой такой толщины необходимо удалить, поскольку при переносе образца из установки ионного травления в магнетронную установку на поверхности образца адсорбируются атомы кислорода и углерода. После удаления слоя образец перемещали в камеру металлов и проводили магнетронное напыление слоев $\mathrm{Co}_{90} \mathrm{Fe}_{10}$ и Та. Магнитное поле при напылении было параллельно направлению оси однонаправленной анизотропии, сформированной при термомагнитной обработке исходного образца $\mathrm{Al}_{2} \mathrm{O}_{3} / \mathrm{Ni}_{77} \mathrm{Fe}_{23}(5 \mathrm{HM}) / \mathrm{Mn}(50 \mathrm{Hм}) / \mathrm{Ni}_{77} \mathrm{Fe}_{23}$ $(30 \mathrm{Hм}) / \mathrm{Ta}(5 \mathrm{Hм})$.

После магнетронного напыления образец $\mathrm{Al}_{2} \mathrm{O}_{3} / \mathrm{Ni}-\mathrm{Fe}-\mathrm{Mn} / \mathrm{Co}_{90} \mathrm{Fe}_{10}(10 \mathrm{Hм}) / \mathrm{Ta}(5 \mathrm{Hм})$ отжигался в магнитном поле при температуре $T_{\text {отж }}=300{ }^{\circ} \mathrm{C}$ в течение 15 мин $\left(T_{\text {отж }}=300{ }^{\circ} \mathrm{C}\right)$, что выше $T_{\mathrm{b}}=270{ }^{\circ} \mathrm{C}$ для $\mathrm{Al}_{2} \mathrm{O}_{3} / \mathrm{Ni}_{77} \mathrm{Fe}_{23}(5 \mathrm{Hм}) / \mathrm{Mn}(50 \mathrm{нм}) / \mathrm{Ni}_{77} \mathrm{Fe}_{23}(30 \mathrm{нм}) / \mathrm{Ta}(5 \mathrm{нм})$. На рис. 2 показаны петли магнитного гистерезиса образца $\mathrm{Al}_{2} \mathrm{O}_{3} / \mathrm{Ni}-\mathrm{Fe}-\mathrm{Mn} / \mathrm{Co}_{90} \mathrm{Fe}_{10}(10 \mathrm{Hм}) / \mathrm{Ta}(5 \mathrm{Hм})$. Продолжительность ионного травления перед магнетронным напылением $\mathrm{Co}_{90} \mathrm{Fe}_{10}$ и Та составляла 20 и 30 мин. 


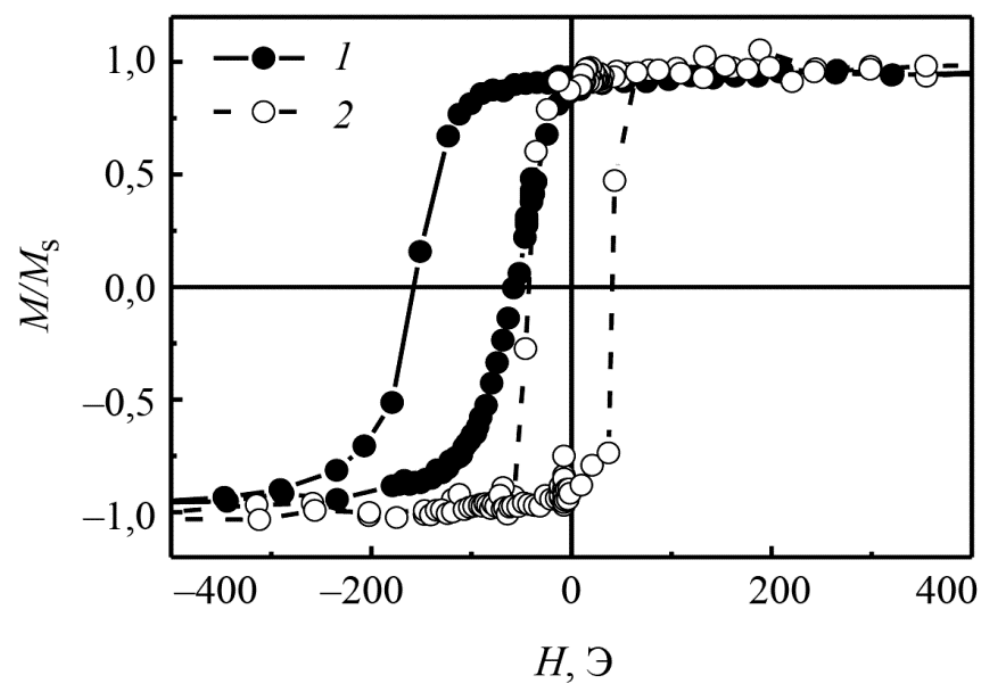

Рис. 2. Петли магнитного гистерезиса $\mathrm{Al}_{2} \mathrm{O}_{3} / \mathrm{Ni}-\mathrm{Fe}-\mathrm{Mn} / \mathrm{Co}_{90} \mathrm{Fe}_{10}(10$ нм $) / \mathrm{Ta}(5$ нм$)$. Ионное травление в течение 20 мин -1 и 30 мин -2

Как видно из рис. 2, смещенная петля гистерезиса наблюдается только в случае травления отожженного образца в течение 20 мин. Сдвиг петли гистерезиса составляет $H_{\mathrm{ex}}=100$ Э, а коэрцитивная сила $H_{\mathrm{c}}=50$ Э. При этом после травления между напыленным слоем $\mathrm{Co}_{90} \mathrm{Fe}_{10}$ и ФМ областью образца существует прямая обменная связь, приводящая к перемагничиванию их как единого целого. При увеличении продолжительности травления до 30 мин $H_{\mathrm{ex}}=0$ Э из-за отсутствия в отожженном образце ФМ области и упорядоченной АФ фазы $\mathrm{Ni}-\mathrm{Fe}-\mathrm{Mn}$.

Таким образом, установлено, что для приготовления спинового клапана с нижним расположением упорядоченной АФ фазы $\mathrm{Ni}-\mathrm{Fe}-\mathrm{Mn}$ необходимо использовать ионное травление отожженного образца $\mathrm{Al}_{2} \mathrm{O}_{3} / \mathrm{Ni}_{77} \mathrm{Fe}_{23}(5 \mathrm{Hм}) / \mathrm{Mn}(50 \mathrm{нм}) / \mathrm{Ni}_{77} \mathrm{Fe}_{23}(30 \mathrm{нм}) / \mathrm{Ta}(5$ нм $)$ продолжительностью 20 мин.

Полученные результаты позволили разработать технологический цикл приготовления наноструктуры типа «спиновый клапан» с нижним расположением АФ упорядоченной фазы $\mathrm{Ni}-\mathrm{Fe}-\mathrm{Mn}$ со следующими операциями:

- формирование упорядоченной АФ фазы $\mathrm{Ni}-\mathrm{Fe}-\mathrm{Mn}$ в образце $\mathrm{Al}_{2} \mathrm{O}_{3} / \mathrm{Ni}_{77} \mathrm{Fe}_{23}(5$ нм)/ $\mathrm{Mn}(50 \mathrm{Hм}) / \mathrm{Ni}_{77} \mathrm{Fe}_{23}(30$ нм $) / \mathrm{Ta}(5$ нм $)$ путем термомагнитной обработки при $260{ }^{\circ} \mathrm{C}$ в течение 4 ч;

- ионное травление в течение 20 мин образца $\mathrm{Al}_{2} \mathrm{O}_{3} / \mathrm{Ni}_{77} \mathrm{Fe}_{23}(5 \mathrm{Hм}) / \mathrm{Mn}(50 \mathrm{Hм}) / \mathrm{Ni}_{77} \mathrm{Fe}_{23}$ (30 нм)/Та(5 нм) для удаления поверхностного слоя;

- магнетронное напыление на подготовленный образец $\mathrm{Al}_{2} \mathrm{O}_{3} / \mathrm{Ni}-\mathrm{Fe}-\mathrm{Mn}$ слоистой структуры, состоящей из ферромагнитных слоев $\mathrm{Co}_{90} \mathrm{Fe}_{10}$, разделенных $\mathrm{Cu}$;

- отжиг приготовленного образца в магнитном поле выше температуры блокировки в течение 15 мин.

На рис. 3 показана полевая зависимость магнитосопротивления приготовленного спинового клапана $\mathrm{Al}_{2} \mathrm{O}_{3} / \mathrm{Ni}-\mathrm{Fe}-\mathrm{Mn} / \mathrm{Co}_{90} \mathrm{Fe}_{10}(5,5 \mathrm{Hм}) / \mathrm{Cu}(3,6 \mathrm{нм}) / \mathrm{Co}_{90} \mathrm{Fe}_{10}(5,5 \mathrm{нм}) / \mathrm{Ta}(5 \mathrm{нм})$ после отжига в магнитном поле при температуре $300{ }^{\circ} \mathrm{C}$ в течение 15 мин. Магнитосопротивление такой структуры составляет $\Delta R / R_{\mathrm{S}} \sim 4 \%$. Это значение существенно выше величины эффекта, полученного в [7], поскольку замена пермаллоя в свободном и пиннингованном слоях на Со или сплав $\mathrm{Co}_{90} \mathrm{Fe}_{10}$ приводит к увеличению спин-зависимого рассеяния и возрастанию магниторезистивного эффекта в спиновом клапане [3]. 


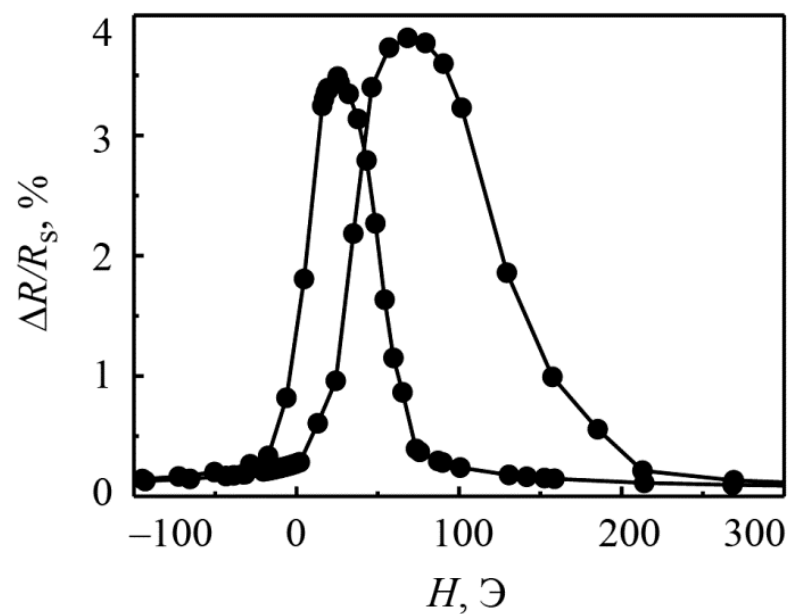

Рис. 3. Полевая зависимость магнитосопротивления спинового клапана $\mathrm{Al}_{2} \mathrm{O}_{3} / \mathrm{Ni}-\mathrm{Fe}-\mathrm{Mn} / \mathrm{Co}_{90} \mathrm{Fe}_{10}(5,5 \mathrm{Hм}) / \mathrm{Cu}(3,6 \mathrm{Hм}) / \mathrm{Co}_{90} \mathrm{Fe}_{10}(5,5 \mathrm{Hм}) / \mathrm{Ta}(5 \mathrm{Hм})$

\section{4. Заключение}

Разработана оригинальная методика создания наноструктур типа «спиновый клапан» с нижним расположением упорядоченной антиферромагнитной фазы $\mathrm{Ni}-\mathrm{Fe}-\mathrm{Mn}$, обладающей высокой температурной стабильностью. Создан спиновый клапан с гигантским магниторезистивным эффектом (около 4 \%), что значительно превышает аналогичный эффект для известных структур такого типа.

\section{Благодарность}

Работа выполнена в рамках государственного задания по теме «Спин» № 01201463330 при поддержке программы фундаментальных исследований УрО РАН (проект № 15-9-2-44) и РФФИ (проект № 16-32-00128).

\section{Список литературы}

1. Ennen I., Kappe D., Rempel T., Glenske C., Hotten A. Giant Magnetoresistance: Basic Concepts, Microstructure, Magnetic Interactions and Applications. Sensors, 2016, vol. 16, no. 6, pp. 904. DOI: $10.3390 / \mathrm{s} 16060904$

2. Baibich M.N., Broto J.M., Fert A., Nguyen van Dau F., Petroff F., Etienne P., Creuzet G., Friederich A., Chazelas J. Giant magnetoresistance of $(001) \mathrm{Fe} /(001) \mathrm{Cr}$ magnetic superlattices. Phys. Rev. Lett., 1988, vol. 61, no. 7, pp. 2472-2475. DOI: 10.1103/PhysRevLett.61.2472

3. Coehoorn R. Giant Magnetoresistance and Magnetic Interactions in Exchange-Biased SpinValves. In: K.H.J. Buschow, ed. Handbook of magnetic materials, Elsevier B.V., Amsterdam, 2003, vol. 15, pp. 1-199.

4. $\quad$ Freitas P.P., Ferreira R., Cardoso S., Cardoso F. Magnetoresistive sensors. J. Phys.: Condens. Matt., 2007, vol. 19, no. 16, pp. 165221-1-21. DOI: 10.1088/0953-8984/19/16/165221

5. Ustinov V.V., Milyaev M.A., Naumova L.I., Giant magnetoresistance of metallic exchangecoupled multilayers and spin Valves. Physics of Metals and Metallography, 2017, vol. 118, no. 13, pp. 1300-1359.

6. $\quad$ Blinov I.V., Krinitsyna T.P., Korolev A.V., Matveev S.A., Arkhipova N.K., Milyaev M.A., Popov V.V., Ustinov V.V. Formation of Ordered NiFeMn Antiferromagnetic Phase in Permalloy/Manganese Bilayers in the Course of Thermomagnetic Treatment. Physics of Metals and Metallography, 2014, vol. 115, pp. 335-341. DOI: 10.1134/S0031918X14040036

7. Svalov A.V., Vas'kovskii V.O., Yarmoshenko Yu.M. Production and study of spin valve structures on the permalloy film base Fiz. Met. Metalloved, 1995, no. 79, pp. 270-273. (In Russian). 